\title{
Lattice QCD Impact on Determination of CKM Matrix: Status and Prospects
}

\section{Steven Gottlieb*}

Indiana University, Bloomington, IN 47405, USA

E-mail: sg@iu.edu

Lattice QCD is an important tool for theoretical input for flavor physics. There have been four reviews by the Flavour Lattice Averaging Group (FLAG). This talk will review the current status of the magnitude of eight of the nine CKM matrix elements, borrowing heavily from the most recent FLAG review (co-authored by the speaker). Future prospects for improving the determination of the CKM matrix will be discussed.

37th International Symposium on Lattice Field Theory - Lattice2019

16-22 June 2019

Wuhan, China

${ }^{*}$ Speaker. 


\section{Introduction}

I was asked to "review very recent FLAG results on standard model parameters and renormalization." This is a very broad charge and one that is ill-suited to the amount of time available, so I will restrict my attention to results from lattice QCD calculations that have an impact on the determination of the CKM mixing matrix. Even with this restriction, it will be necessary to summarize results at a rather high level. Many details of the calculations can be found in the latest and recent FLAG reviews [1, 2], and, of course, the original papers cited therein.

The Cabibbo-Kobayashi-Maskawa (CKM) mixing matrix is fundamental to the field of flavor physics within the Standard Model (SM) of Elementary Particle and Nuclear Physics. Kobayashi and Maskawa were awarded the Nobel Prize for their realization that with three generations of quarks the matrix may contain a complex phase that results in $C P$ violation. Although it seems that this is not sufficient to explain the baryon asymmetry of the universe, there are many processes in which to test the CKM scenario, and any such tests in which the SM fails to explain the observations could give a window into new physics beyond the standard model (BSM).

A few words about my background may be in order. I am a founding member of the MILC Collaboration and a member of the Fermilab Lattice/MILC effort that is some 16 years old. I am also a member of the Flavour Lattice Averaging Group (FLAG) where I have been working in the $B$ and $D$ semileptonic working group. However, this is not a FLAG approved talk, so I am solely responsible for its content. The two most recent editions of the FLAG report are in Refs. [1] and [2]. I will use many plots from the most recent FLAG report and cover results from several of the working groups. I will also include several graphs from the Fermilab Lattice/MILC Collaborations. I am grateful for the work of all my FLAG, Fermilab Lattice, and MILC collaborators.

\section{CKM Matrix}

The CKM matrix describes how quarks mix under the weak interaction, that is, the misalignment of mass eigenstates and weak eigenstates. The CKM matrix is shown in expression 2.1. The matrix elements are shown in bold type, and beneath the elements in the first two rows you will find one or two weak decays that can be used to determine that matrix element, if we can accurately calculate the QCD contribution to the decay. Under the last row of elements are two hadronic matrix elements that remind us that $B_{(s)}$ mixing allows us to determine $V_{t d}$ and $V_{t s}$.

$$
\left(\begin{array}{ccc}
\mathbf{V}_{\mathbf{u d}} & \mathbf{V}_{\mathbf{u s}} & \mathbf{V}_{\mathbf{u b}} \\
\pi \rightarrow l v & K \rightarrow l v & B \rightarrow l v \\
& K \rightarrow \pi l v & B \rightarrow \pi l v \\
\mathbf{V}_{\mathbf{c d}} & \mathbf{V}_{\mathbf{c s}} & \mathbf{V}_{\mathbf{c b}} \\
D \rightarrow \pi l v & D \rightarrow K l v & B \rightarrow D^{(*)} l v \\
D \rightarrow l v & D_{s} \rightarrow l v & \Lambda_{b} \rightarrow \Lambda_{c} l v \\
\mathbf{V}_{\mathbf{t d}} & \mathbf{V}_{\mathbf{t s}} & \mathbf{V}_{\mathbf{t b}} \\
\left\langle B_{d} \mid \bar{B}_{d}\right\rangle & \left\langle B_{s} \mid \bar{B}_{S}\right\rangle &
\end{array}\right)
$$

The CKM matrix is unitary so each row and each matrix is a complex unit vector. Each row (column) is orthogonal to the other two rows (columns). Violations of unitarity are evidence of 
BSM physics. It is important to use multiple processes to determine each matrix element. If two different processes infer different values for the same CKM matrix element, that would also be evidence for non-standard model physics. Of course, we would very much like to have solid evidence for BSM physics, but that requires precise determination of the standard model contribution. Lattice QCD is one of the best tools for calculating those contributions.

As a first example, let's consider the leptonic branching fraction for the $D_{(s)}$ meson:

$$
\mathscr{B}\left(D_{(s)} \rightarrow \ell v_{\ell}\right)=\frac{G_{F}^{2}\left|V_{c q}\right|^{2} \tau_{D_{(s)}}}{8 \pi} f_{D_{(s)}}^{2} m_{\ell}^{2} m_{D_{(s)}}\left(1-\frac{m_{\ell}^{2}}{m_{D_{(s)}}^{2}}\right)^{2} .
$$

On the right hand side, the Fermi constant, lepton mass, meson mass, and meson lifetime appear. These are all well determined. Also appearing are the CKM matrix element $V_{c q}$ that we would like to determine, and the so-called hadronic decay constant $f_{D_{(s)}}$ that we calculate using lattice QCD. A second example is a semileptonic $D$ meson decay for which the differential decay rate may be written:

$$
\begin{array}{r}
\frac{d \Gamma(D \rightarrow P \ell v)}{d q^{2}}=\frac{G_{\mathrm{F}}^{2}\left|V_{c x}\right|^{2}}{24 \pi^{3}} \frac{\left(q^{2}-m_{\ell}^{2}\right)^{2} \sqrt{E_{P}^{2}-m_{P}^{2}}}{q^{4} m_{D}^{2}}\left[\left(1+\frac{m_{\ell}^{2}}{2 q^{2}}\right) m_{D}^{2}\left(E_{P}^{2}-m_{P}^{2}\right)\left|f_{+}\left(q^{2}\right)\right|^{2}\right. \\
\left.+\frac{3 m_{\ell}^{2}}{8 q^{2}}\left(m_{D}^{2}-m_{P}^{2}\right)^{2}\left|f_{0}\left(q^{2}\right)\right|^{2}\right]
\end{array}
$$

where $x=d, s$ is the daughter light quark, $P=\pi, K$ is the daughter light-pseudoscalar meson, $q=\left(p_{D}-p_{P}\right)$ is the momentum of the outgoing lepton pair, and $E_{P}$ is the light-pseudoscalar meson energy in the rest frame of the decaying $D$. A similar formula holds for other heavy-light mesons such a $D_{s}, B$ or $B_{s}$. The hadronic physics that we require is expressed in terms of the two form factors $f_{+}\left(q^{2}\right)$ and $f_{0}\left(q^{2}\right)$. They are defined in terms of the hadronic matrix element of the flavor-changing vector current $V_{\mu}=\bar{x} \gamma_{\mu} c$,

$$
\left\langle P\left|V_{\mu}\right| D\right\rangle=f_{+}\left(q^{2}\right)\left(p_{D \mu}+p_{P \mu}-\frac{m_{D}^{2}-m_{P}^{2}}{q^{2}} q_{\mu}\right)+f_{0}\left(q^{2}\right) \frac{m_{D}^{2}-m_{P}^{2}}{q^{2}} q_{\mu} .
$$

The experimental observable depends upon known quantities, a CKM matrix element, and the hadronic information that is encoded the form factors which depend upon $q^{2}$.

\section{First Row}

Because $\left|V_{u b}\right|$ is so small, we can test unitarity of the first row of the CKM matrix quite well (with current precision) looking only at pion and kaon leptonic decays and kaon semileptonic decay. Figure 1 summarizes results for $f_{\pi}$ and $f_{K}$, the pion and kaon decay constants, respectively. This is the first of a number of plots from FLAG [2], so we should explain the color coding. Solid green symbols correspond to calculations for which there should be sufficient control of systematic errors. There are criteria for lattice volume, quark masses (to control the chiral limit) and number of lattice spacings (to control the continuum limit). Some quantities have additional quality criteria detailed in the FLAG report [2]. Points in green with open plotting symbols have been superseded, usually because a group has added additional ensembles or statistics. Points plotted in red are 


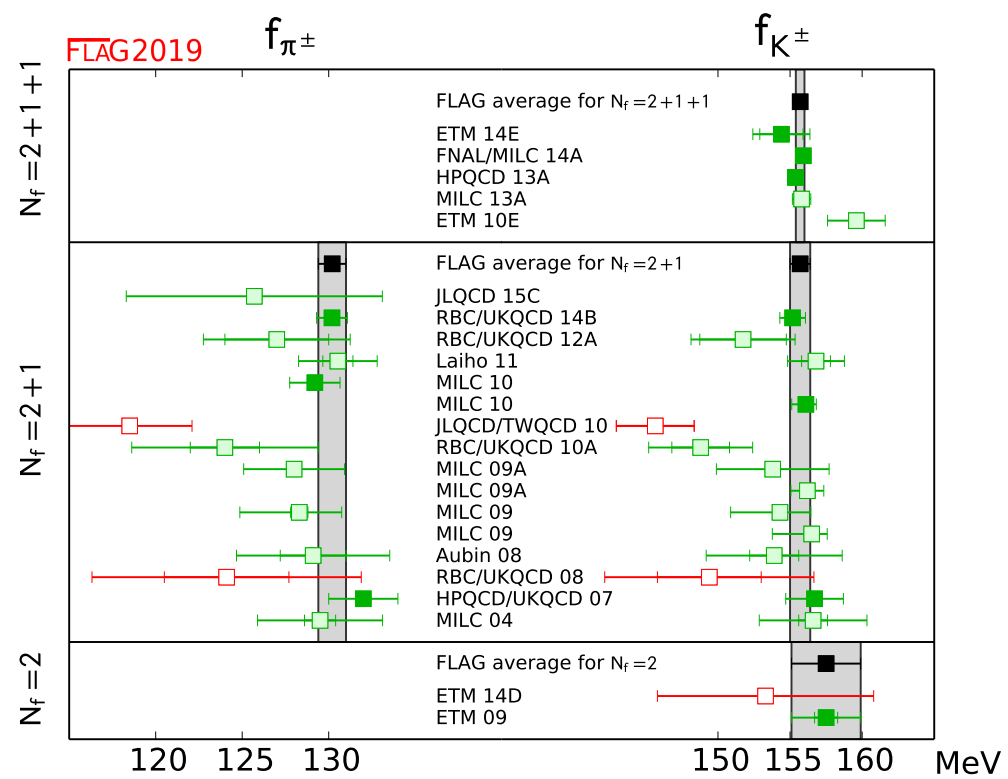

Figure 1: FLAG 2019 compilation of results for $f_{\pi}$ and $f_{K}$. Meaning of the colors is explained the the text.

deemed not to have adequate control of systematic errors. The black points and gray bands are the FLAG average values. Only solid green points are included in the FLAG average values. Each graph is generally divided into sections depending upon the number of dynamical quarks in the sea. The values $N_{f}=2,2+1$, and $2+1+1$ are used for two-light flavors, two light plus strange, and two light plus strange plus charm, respectively. In most cases, there is a FLAG average for each value of $N_{f}$. We will restrict our attention to $N_{f}=2+1$ and $2+1+1$.

In Fig. 1 , one notices that in many cases there is a value of $f_{K}$, but none for $f_{\pi}$. This is usually because $f_{\pi}$ has been used to set the scale. Marciano [3] pointed out that the ratio $f_{K} / f_{\pi}$ can be used to determine the ratio $\left|V_{u s} / V_{u d}\right|$ as leptonic decays of both pion and kaon are well measured in experiment. As summarized by the Particle Data Group [4] and Moulson at CKM 2017 [5],

$$
\left|\frac{V_{u s}}{V_{u d}}\right| \frac{f_{K^{ \pm}}}{f_{\pi^{ \pm}}}=0.2760(4) .
$$

Thus, the experimental error is $0.15 \%$. Figures 2 and 3 display summaries of results from multiple groups for the decay constant ratio. Figure 2 is from FLAG [2], while Fig. 3 is from a recent Fermilab/MILC publication [6]. The former is more comprehensive, but the latter uses a finer scale, and one can actually see the error bars on the most precise calculations, as they are no longer obscured by the plotting symbols. The FLAG 2019 result for $N_{f}=2+1+1$ is $1.1932(19)$. The previous FLAG result [1] was 1.193(3). The FNAL/MILC 2017 result is $1.1950\left({ }_{-22}^{+15}\right)$. The theory error has been reduced to $0.16 \%$, quite comparable to the experimental error.

Let us now turn from leptonic decays to the semileptonic decay of the K meson. Since semileptonic decays have three-body final states, the kinematics are slightly more complicated, and there is one kinematic variable usually called $q^{2}$ upon which the form factor depends. From energymomentum conservation, $p_{K}=p_{\pi}+q_{\ell}+q_{\nu}$ where $p_{K}$ is the energy-momentum 4-vector of the 


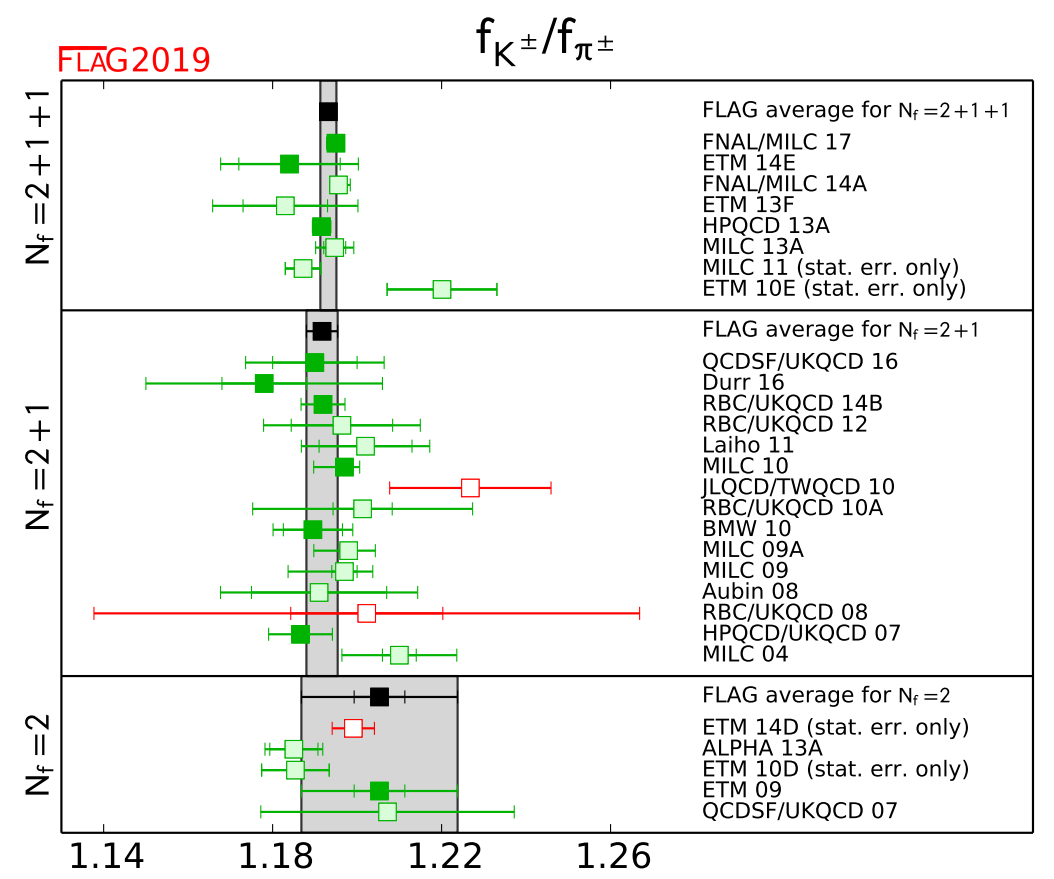

Figure 2: FLAG 2019 compilation of ratio $f_{K} / f_{\pi}$. Comparison of calculations of decay constant ratio $f_{K} / f_{\pi}$ with $N_{f}=2+1+1,2+1$, and 2 sea quark flavors. From Ref. [2].

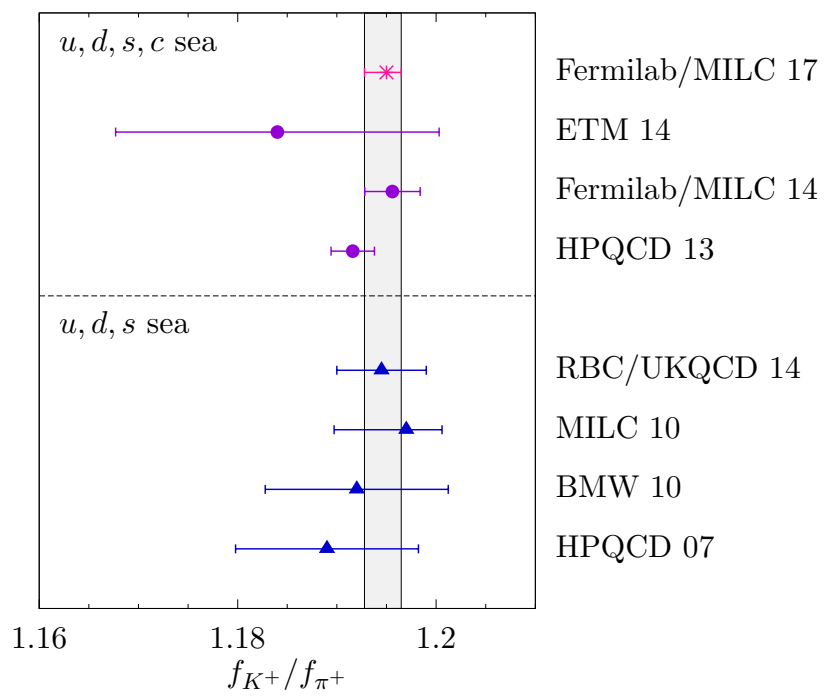

Figure 3: Comparison of calculations of decay constant ratio $f_{K} / f_{\pi}$ with $N_{f}=2+1+1$ and $2+1$ sea quark flavors. From Ref. [6].

initial state kaon, $p_{\pi}$ refers to the final state pion, and $q_{\ell}$ and $q_{v}$, refers to the final state lepton and neutrino. The momentum transferred to the leptons $q=q_{\ell}+q_{v}$, and we have already introduced $q^{2}$.

To determine $\left|V_{u s}\right|$ from experiment, we could determine the vector form factor $f_{+}\left(q^{2}\right)$ and use 


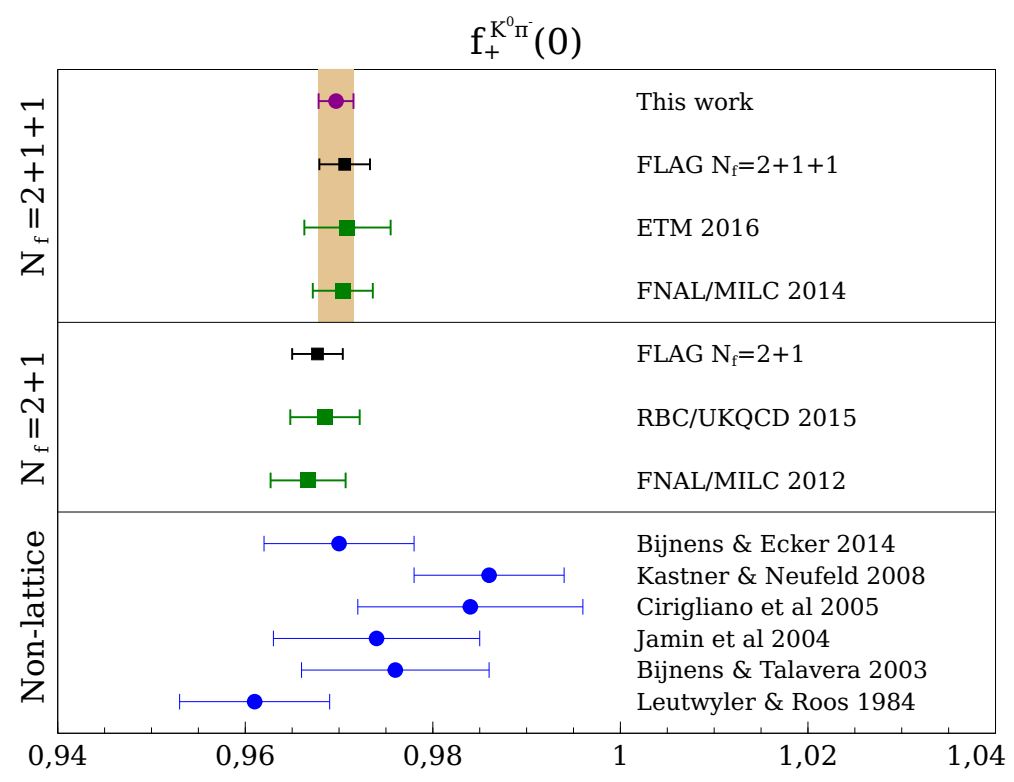

Figure 4: Comparison of calculations of the vector form factor $f_{+}\left(q^{2}=0\right)$ with $N_{f}=2+1+1$ and $2+1$ sea quark flavors, and other non-lattice QCD methods. From Ref. [7].

the differential decay rate of the kaon; however, it is convenient to just calculate $f_{+}\left(q^{2}=0\right)$ and use experimental input that determines $\left|V_{u s}\right| f_{+}(0)$. We would like to start our story in 2014, when the experimental value was $\left|V_{u s}\right| f_{+}(0)=0.2165(4)$. At that time, FNAL/MILC had an $N_{f}=2+1+1$ value $f_{+}(0)=0.9704(24)(22)$ where the first error was statistical and the second systematic. So, in 2014 , the experimental error was $0.18 \%$, but the theory error was $0.34 \%$. Moving ahead to late 2018, the experimental result had been slightly updated [5] to: $\left|V_{u s}\right| f_{+}(0)=0.21654(41)$. Also FNAL/MILC [7] updated their calculation resulting in

$$
f_{+}(0)=0.9696(15)_{\text {stat }}(12)_{\text {sys }}=0.9696(19),
$$

so the theory error is $0.20 \%$, quite comparable to the experimental error. The FLAG 2019 average for $N_{f}=2+1+1$ is $f_{+}(0)=0.9706(27)$. The FLAG result has a larger error because Ref. [7] had not been published before the FLAG deadline for inclusion in the latest edition. Figure 4, taken from Ref. [7], depicts the most relevant lattice calculations for $N_{f}=2+1$ and $2+1+1$. It also contains results from a number of other theoretical approaches whose errors are larger than those from lattice QCD.

We now turn to the $V_{u d}-V_{u s}$ plane to consider the implications of what we have just found. The ratio of decay constant determines the ratio $\left|V_{u s} / V_{u d}\right|$ and thus an angled band in the plane of the two CKM matrix elements. The semileptonic kaon decay determines a horizontal band for $\left|V_{u s}\right|$. Nuclear $\beta$-decay provides a fairly precise value of $\left|V_{u d}\right|$, i.e., a vertical band. In addition, because $\left|V_{u b}\right|$ is so small, unitarity determines a very narrow arc of a circle in the plane. Figure 5 describes the situation according to FLAG [2]. We see that the two white ellipses that come from the intersection of leptonic and semileptonic decay bands show some tension with both unitarity and $V_{u b}$ from $\beta$-decay. This graph has results for both $N_{f}=2+1$ and $2+1+1$ lattice QCD 


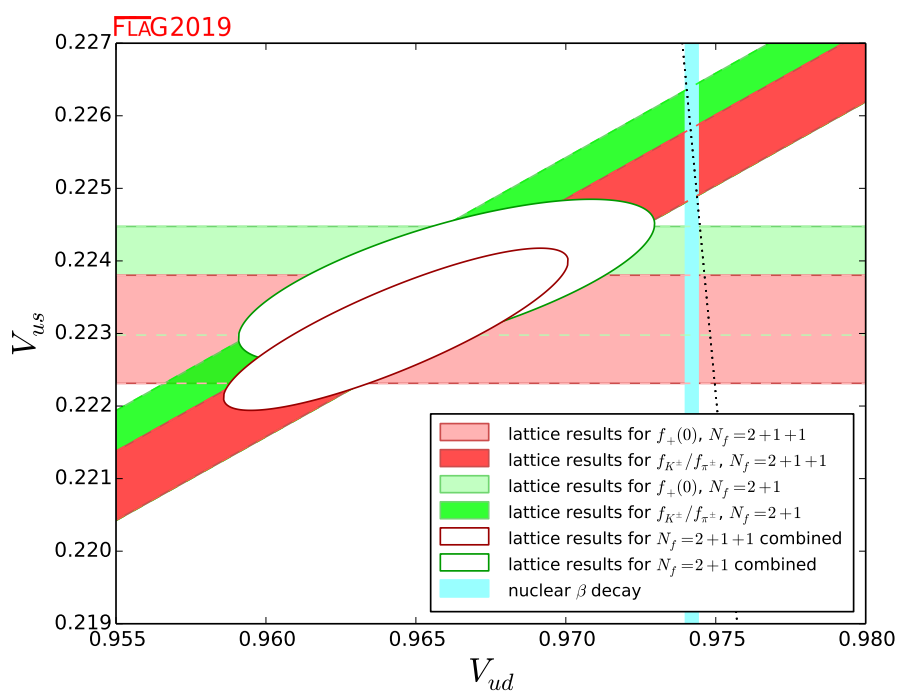

Figure 5: First row unitarity plot from FLAG [2].

calculations. It should be noted that the decay constant ratio is in reasonable agreement with $\beta$ decay and unitarity. In Fig. 6, we show the results from Ref. [7]. In this case, we have $N_{f}=2+1+1$ and both blue ellipses show tension with unitarity. The narrow horizontal band is labeled $K_{\ell 3}$, a common notation for kaon semileptonic decay, while the angled band is labeled $K_{\ell 2}$ a notation for kaon leptonic decay. In addition, there is a wide horizontal band which is determined from the known value of $\left|V_{c d}\right|$ and the assumption of CKM unitarity. (Recall that in the Wolfenstein representation, $V_{c d}=-V_{u s}$.)

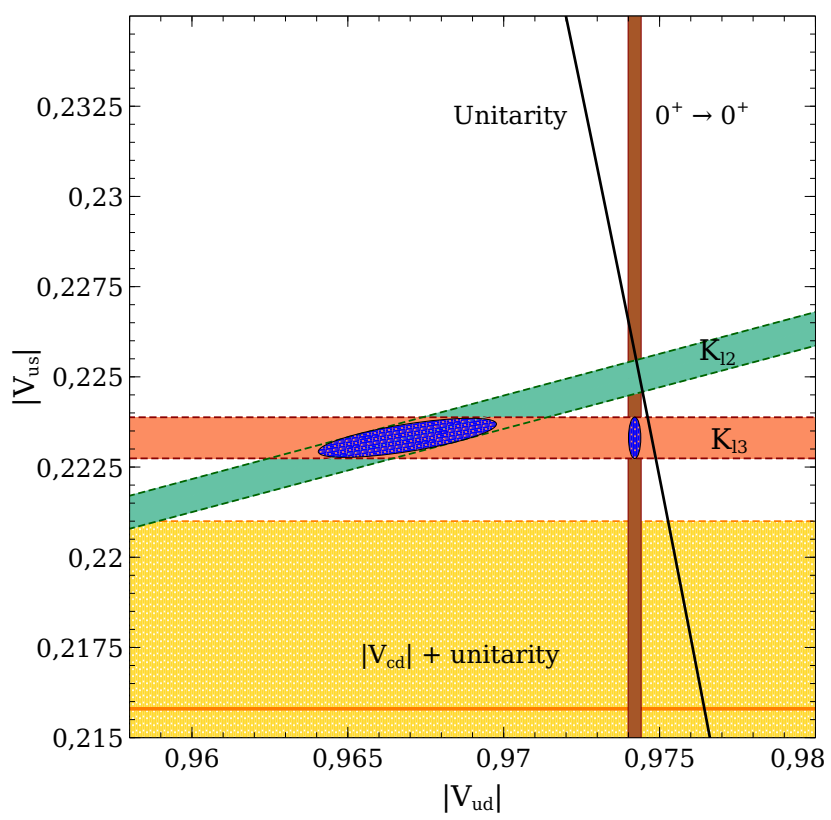

Figure 6: First row unitarity plot from FNAL/MILC [7]. 


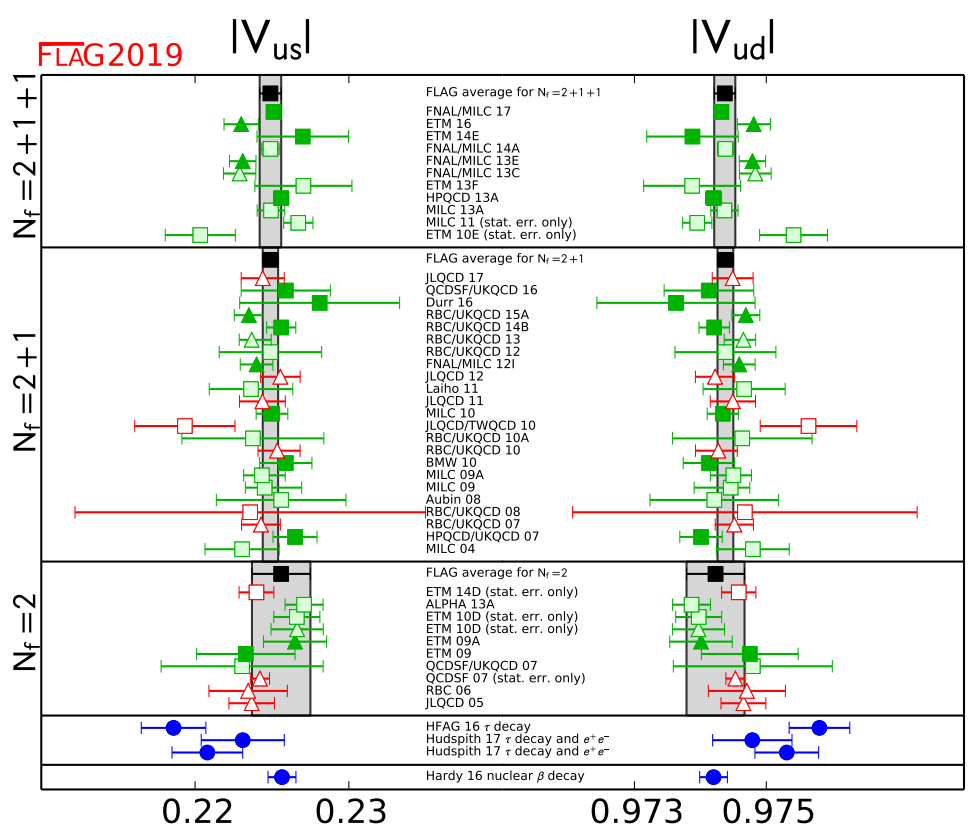

Figure 7: Determination of $\left|V_{u s}\right|$ and $\left|V_{u d}\right|$ from leptonic and semileptonic decays from FLAG [2]. Squares denote results from leptonic decays and triangles are used for semileptonic decays.

We have a summary of determination of $\left|V_{u s}\right|$ and $\left|V_{u d}\right|$ from FLAG in Fig. 7. This plot assumes unitarity to go from either band to a value. Squares denote values from leptonic decays, and triangles are used for semileptonic decays. The tension we saw in the previous two plots is seen as a difference between triangles and squares, which is most noticeable for $N_{f}=2+1+1$. FLAG results for $N_{f}=2+1+1$ are:

$$
\begin{aligned}
& \left|V_{u s}\right|=0.2249(7) \\
& \left|V_{u d}\right|=0.97437(16)
\end{aligned}
$$

The blue values near the bottom of the plot show results based on standard model analysis of $\tau$ decay and nuclear $\beta$-decay. Please see the FLAG report [2] for references.

\section{Second Row}

Returning to expression 2.1, we find several semileptonic decays on the row below $\mathbf{V}_{\mathbf{c d}}$, and leptonic decays of $D$ and $D_{s}$ on the following row. We will consider the leptonic decays first.

It has been almost 15 years since the initial test of $D$ meson decay constants at CLEO-c [8]. The unquenched calculations were based on $N_{f}=2+1$ ensembles with asqtad quarks [9]. Those early measurements and calculations had errors of about $8 \%$. By 2014, the theoretical error had fallen by more than a factor of ten. In 2017, using $N_{f}=2+1+1$ ensembles with HISQ quarks, errors on the charm decay constants have dropped below $0.3 \%$, considerably below the precision of results with $N_{f}=2+1$. Figure 8 from Ref. [6] depicts several recent calculations of the $D_{(s)}$ 


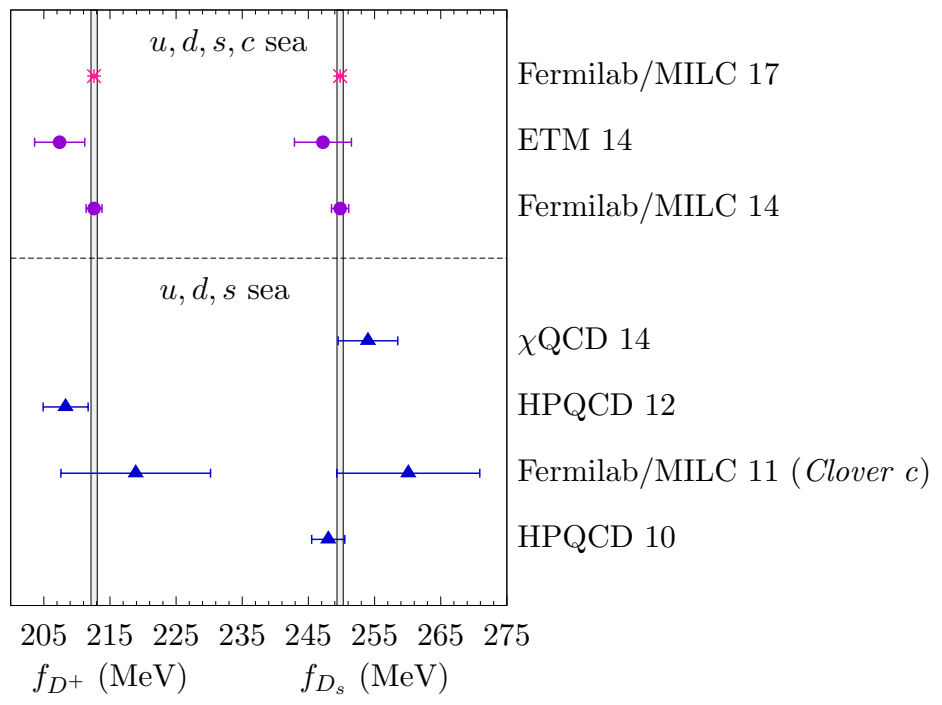

Figure 8: Comparison of recent calculations of $f_{D^{+}}$and $f_{D_{s}}$ with $N+f=2+1+1$ and $2+1$ [6].

meson decay constants. The interested reader can find a more complete set of values in Ref. [2]. The results in Ref. [6] are:

$$
f_{D^{+}}=212.7(0.6) \mathrm{MeV}, \quad f_{D_{s}}=249.9(0.4) \mathrm{MeV} .
$$

The Particle Data Group [4] has compiled the results of several experiments to provide the best values for the product of decay constant and CKM matrix elements. The experimental values are: $f_{D}\left|V_{c d}\right|=45.91(1.05) \mathrm{MeV}$ and $f_{D_{s}}\left|V_{c s}\right|=250.9(4.0) \mathrm{MeV}$. These values have errors of 1.6$2.3 \%$, so the experimental error is going to be dominant in determination of the two CKM matrix elements. From Ref. [6], we have

$$
\begin{aligned}
\left|V_{c d}\right|_{\mathrm{SM}, f_{D}} & =0.2152(5)_{f_{D}}(49)_{\text {expt }}(6)_{\mathrm{EM}}, \\
\left|V_{c s}\right|_{\mathrm{SM}, f_{D_{S}}} & =1.001(2)_{f_{D_{s}}}(16)_{\text {expt }}(3)_{\mathrm{EM}},
\end{aligned}
$$

where the errors are from lattice decay constant, experiment, and a structure dependent electromagnetic correction. These values differ slightly from those in FLAG [2]. Earlier this year, some new results for $D_{s}$ decay were published by BESIII [10]. It was found that $f_{D_{s}}\left|V_{c S}\right|=246.2(5.0) \mathrm{MeV}$. This will bring the world average down and help improve the second row unitary sum. In fact, after Lattice 2019, the Heavy Flavor Averaging Group (HFLAV)) [11] updated its results for many quantities of interest including the leptonic decays of $D$ and $D_{s}$ mesons. Their current world averages are $f_{D}\left|V_{c d}\right|=46.1(1.1) \mathrm{MeV}$ and $f_{D_{s}}\left|V_{c s}\right|=247.8(3.1) \mathrm{MeV}$. These errors are 2.4 and $1.3 \%$, respectively, which is an improvement for $D_{s}$. HFLAV uses the FLAG results for the decay constants, which have slightly more generous errors than those in Ref. [6]. The FLAG value of $f_{D}$ is also slightly smaller. With that input, HFLAV finds $\left|V_{c d}\right|=0.2173(51)_{\text {expt }}(7)_{L Q C D}$ and $\left|V_{c s}\right|=0.991(13)_{\text {expt }}(2)_{L Q C D}$.

For charm semileptonic decays, a single form factor describes the major contribution to the decay rate. The analysis is often restricted to $q^{2}=0$. In that case, we can use the world average 


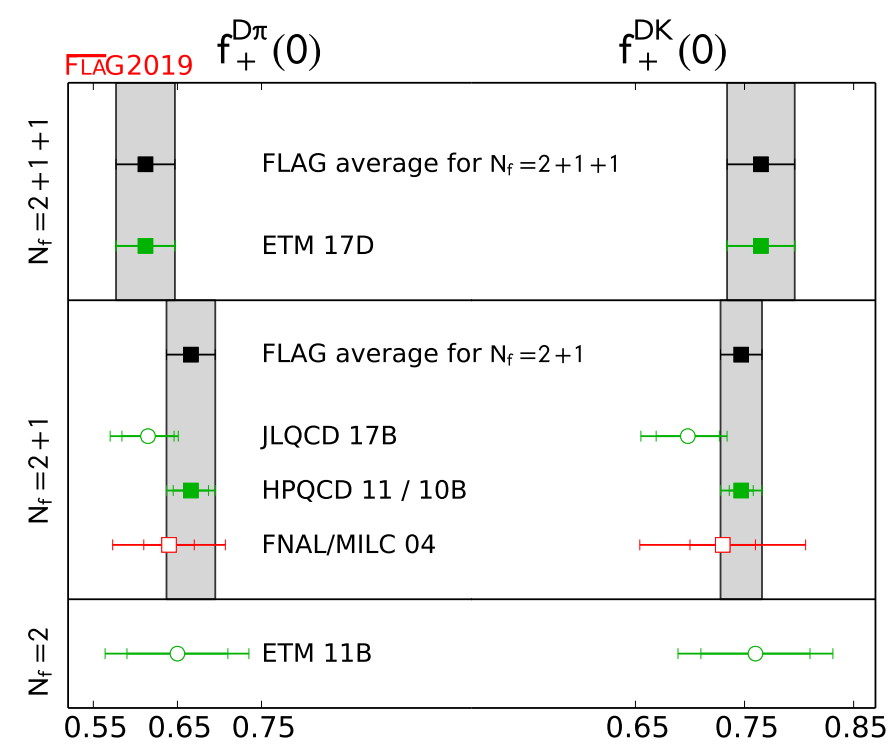

Figure 9: FLAG [2] summary of results for $D$ semileptonic decays to $\pi$ or $K$.

values provided by the Heavy Flavor Averaging Group (HFLAV) [11]: $f_{+}^{D \pi}(0)\left|V_{c d}\right|=0.1426(18)$ and $f_{+}^{D K}(0)\left|V_{c S}\right|=0.7180(33)$. These values have been updated since Lattice 2019 and do not agree with the values quoted in my slides on the conference indico site. In particular, the second value has decreased. The experimental errors are $1.3 \%$ and $0.5 \%$ for the two decays. Figure 9 shows the FLAG averages for both form factors at $q^{2}=0$. For both $N_{f}=2+1$ and $2+1+$ there is only a single result. Consider the form factors for $D \rightarrow K \ell v$, as that has the smaller experimental error, the $N_{f}=2+1+1$ result from ETM [12] is $0.765(31)$, i.e., a error of $4.2 \%$. The $N_{f}=2+1$ result from HPQCD $[13,14]$ is $0.747(19)$ or a $2.5 \%$ result. We see that the theoretical errors need to decrease by a factor of $2-4$ to match the current experimental precision. It should be noted that ETM has form factor results as a function of $q^{2}$. Also, FNAL/MILC are expecting to have a result with an error of about $2.1 \%$. FLAG had summarized the results for $\left|V_{c d}\right|$ and $\left|V_{c s}\right|$ which are displayed in Fig. 10. As before, squares are used for results from leptonic decays, and triangles for semileptonic decays. These FLAG results use an older value of $f_{+}^{D K}(0)\left|V_{c s}\right|=0.7226(34)$. The result labeled Meinel 16 is from baryon decay, and the result labeled ETM 17D/Riggio 17 uses non-zero values of $q^{2}$ to extract $\left|V_{c s}\right|$ from the differential decay rate. Experimental errors dominate for the leptonic decays, while the theory error is dominant for semileptonic decays. Here are some key FLAG results.

leptonic decays, $N_{f}=2+1+1:\left|V_{c d}\right|=0.2166(7)(50),\left|V_{c s}\right|=1.004(2)(16)$,

$$
\text { leptonic decays, } N_{f}=2+1:\left|V_{c d}\right|=0.2197(25)(50),\left|V_{c s}\right|=1.012(7)(16) \text {, }
$$

semileptonic decays, $N_{f}=2+1+1: \quad\left|V_{c d}\right|=0.2341(74), \quad\left|V_{c s}\right|=0.970(33)$,

$$
\text { semileptonic decays, } N_{f}=2+1:\left|V_{c d}\right|=0.2141(93)(29),\left|V_{c s}\right|=0.967(25)(5) \text {, }
$$

semileptonic $\Lambda_{\mathrm{c}}$ decay, $N_{f}=2+1$ :

$\left|V_{c s}\right|=0.949(24)(51)$, 


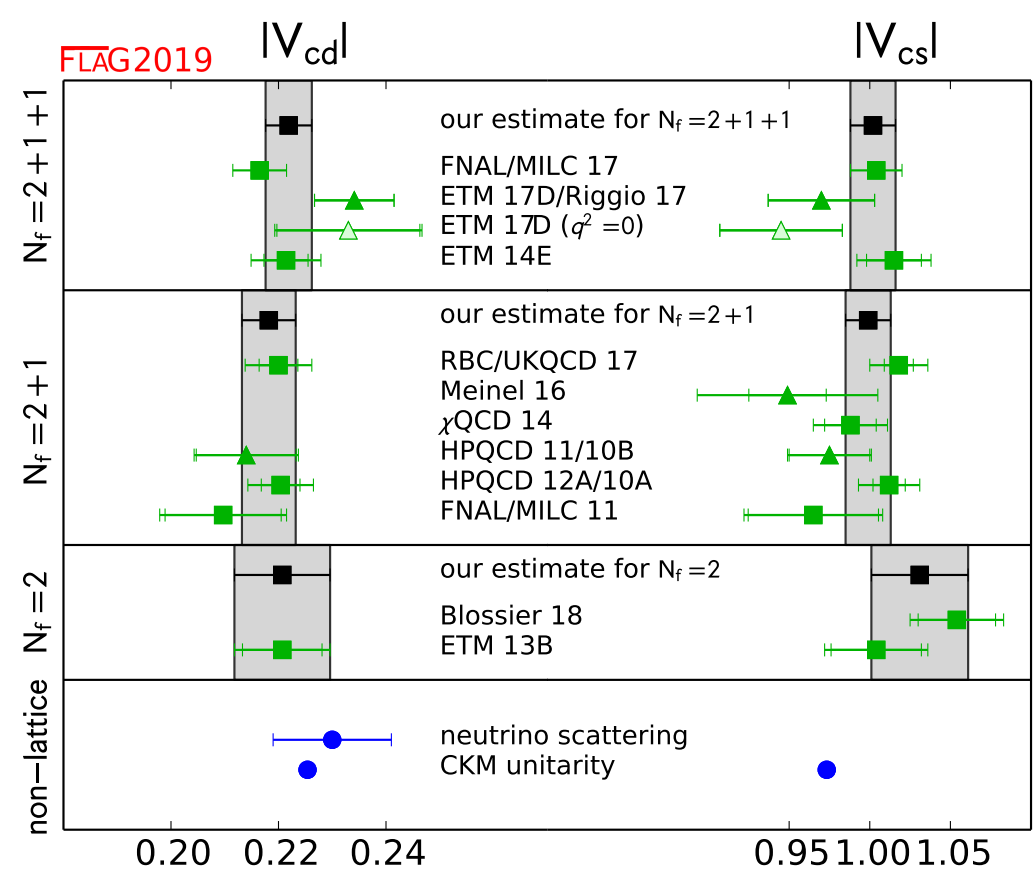

Figure 10: FLAG [2] summary of results for $\left|V_{c d}\right|$ and $\left|V_{c s}\right|$. Squares are used for results from leptonic decays, and triangles for semileptonic decays.

$$
\begin{aligned}
\text { FLAG2019, } N_{f}=2+1+1: & \left|V_{c d}\right|=0.2219(43), & & \left|V_{c s}\right|=1.002(14), \\
\text { FLAG2019, } N_{f}=2+1: & \left|V_{c d}\right|=0.2182(50), & & \left|V_{c s}\right|=0.999(14),
\end{aligned}
$$

Let's consider second row unitarity. Using their most recent leptonic decay results [6] Fermilab/MILC have: $\left|V_{c d}\right|^{2}+\left|V_{c s}\right|^{2}+\left|V_{c b}\right|^{2}=1.049(2)_{\left|V_{c d}\right|}(32)_{\left|V_{c s}\right|}(0)_{\left|V_{c b}\right|}$, which is just over $1.5 \sigma$ from 1. The FLAG 2019 value for $N_{f}=2+1$ and $2+1+1$ is $1.05(3)$, which is similar. The unitarity sum from ETM [15], which is based on semileptonic decays, is 0.996(64). It agrees very well with unitarity, but with a larger error than what is seen from leptonic decays. We had already seen that semileptonic decays favor a smaller value of $\left|V_{c s}\right|$ than leptonic decays do, so the improved agreement with unitarity should not be a surprise. If we consider the latest HFLAV update, we have: $\left|V_{c d}\right|^{2}+\left|V_{c s}\right|^{2}+\left|V_{c b}\right|^{2}=1.029(2)_{\left|V_{c d}\right|}(26)_{\left|V_{c s}\right|}(0)_{\left|V_{c b}\right|}$, which is only about $1.1 \sigma$ away from 1 . Thus, we have reasonable agreement with unitarity for both leptonic and semileptonic charm meson decays, with a few percent accuracy. It will, or course, be interesting to increase the precision of this test in the future.

\section{B Hadron Decays}

Leptonic and semileptonic decays of hadrons containing a $b$ quark have been studied using lattice QCD. Mesonic decays have been extensively studied. Recently, Meinel and his collaborators have been looking at various baryon semileptonic decay form factors [16]. In addition to tree level decays such as $B \rightarrow \pi \ell v$, which can be used to determine $\left|V_{u b}\right|$, there have been studies of rare decays that involve flavor changing neutral currents [17]. Such decays vanish at tree level in the 


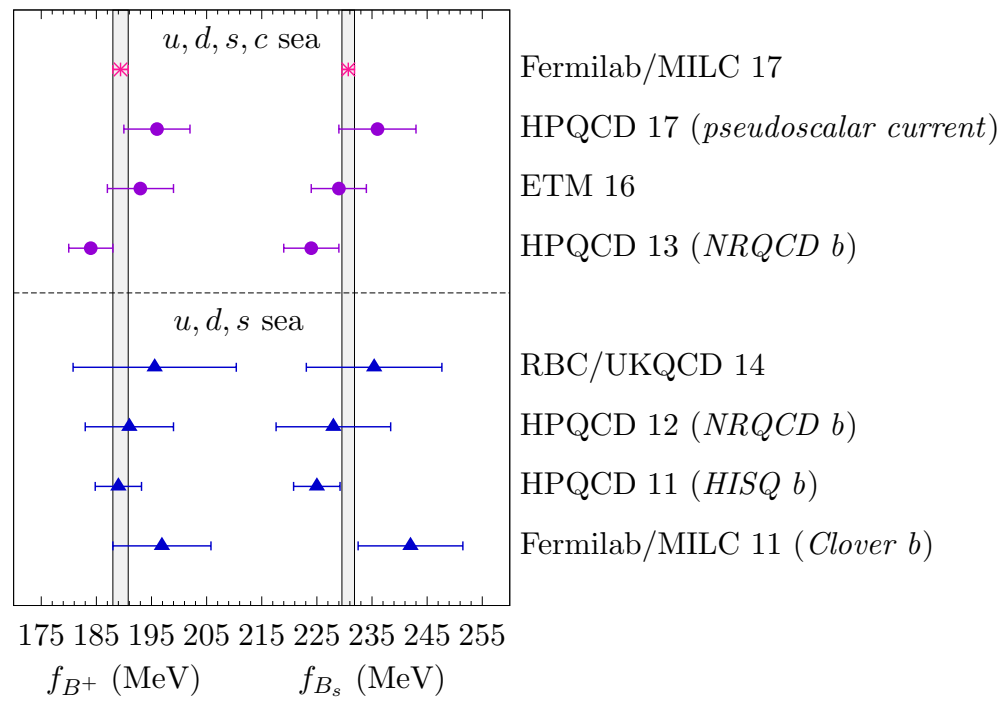

Figure 11: Comparison of recent results for $f_{B^{+}}$and $f_{B_{s}}$ from Ref. [6].

Standard Model, so they are a good place to search for new physics. They provide a complementary method to $B$ mixing studies for determining $\left|V_{t d}\right|$ and $\left|V_{t s}\right|$. There have been some interesting tensions between recent Standard Model predictions and LHCb measurements, including hints of lepton universality violation. (See Ref. [17] for a summary.) Although these issues are very interesting, we will need to stay focused on determination of CKM matrix elements.

In Fig. 11, we show a compilation of results from Ref. [6]. The latest results from FNAL/MILC have errors under $1.3 \mathrm{MeV}$, i.e., $<0.7 \%$. These results are in good agreement with prior results that had errors as small as 5-7 MeV. Unfortunately, the BaBar and Belle experimental measurements of $B$ decay do not agree very well and have large errors, so the determination of $\left|V_{u b}\right|$ is very much limited by the required experimental input. In the next few years, Belle II should provide much higher precision results. Additional details and historical calculations may be found in Refs. [1] and [2].

As mentioned above, $B$ semileptonic and rare decays have been studied both experimentally and theoretically. Several decays such a $B \rightarrow \pi \ell v, B_{s} \rightarrow K^{(*)} \ell v$, and $\Lambda_{b} \rightarrow p \ell v$ all depend on $\left|V_{u b}\right|$. These decays can be used to determine $\left|V_{c b}\right|: B \rightarrow D^{(*)} \ell v, B_{s} \rightarrow D_{s}^{(*)} \ell v$, and $\Lambda_{b} \rightarrow \Lambda_{c} \ell v$. Recall that when a particular CKM matrix element can be determined from multiple decays, if the standard model predictions of the decay rates do not imply identical values of the particular CKM matrix element, that would be evidence for new physics not in the standard model. In addition, since $\ell$ can be an electron, muon or $\tau$, there is an opportunity to test lepton universality. Recently, some tensions with lepton universality have been seen, and this is a fertile area of study. Unfortunately, we do not have time to discuss this in detail. Interesting rare decays include $B^{0} \rightarrow \mu^{+} \mu^{-}, B_{s} \rightarrow$ $\mu^{=} \mu^{-}$, and $B \rightarrow K \ell^{=} \ell^{-}$.

There has been a long standing difference between the values of $\left|V_{u b}\right|$ and $\left|V_{c b}\right|$ as determined from exclusive and inclusive decay results. The history of the comparison from 2009 to 2018 is shown in Fig. 12. When the precision of each determination was low in 2009, there was not a tension between the two values; however, by 2014 the difference had grown. In 2015, the difference 


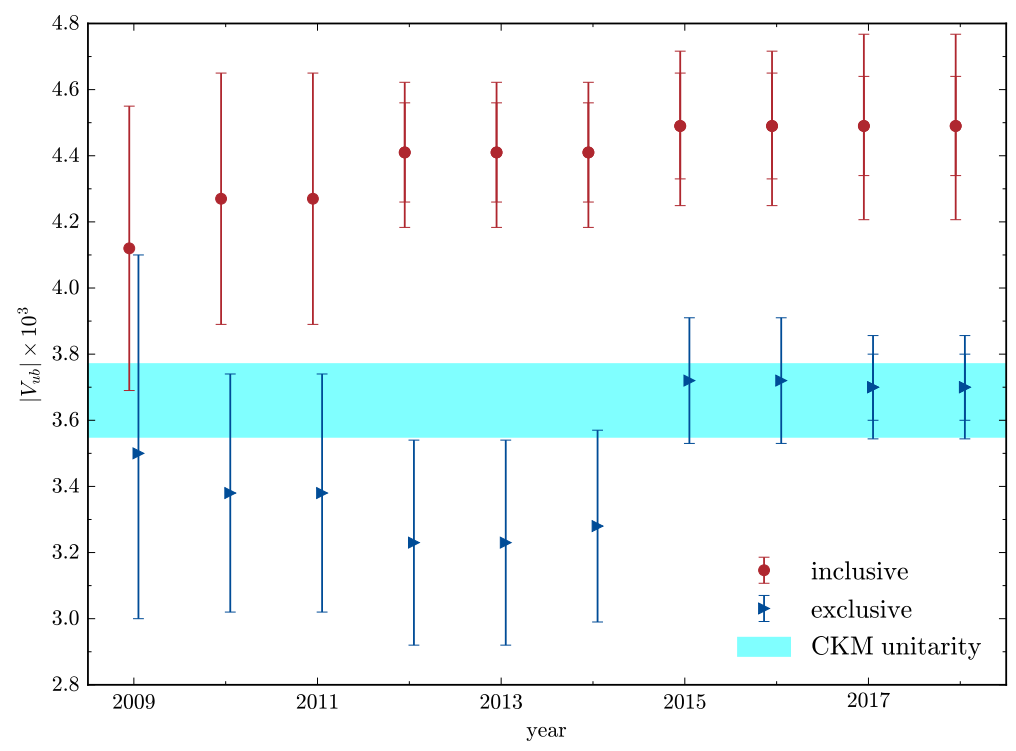

Figure 12: History of $\left|V_{u b}\right|$ as determined from both inclusive and exclusive measurements.

was reduced, but there has not been much change since then. The plot also has a horizontal band corresponding to CKM unitarity.

The change in the value of $\left|V_{u b}\right|$ as determined from exclusive decays resulted from a new form factor calculation from the Fermilab Lattice and MILC collaborations [18]. Experimental results from both $\mathrm{BaBar}$ and Belle were used to determine $\left|V_{u b}\right|=3.72(16) \times 10^{-3}$. Figure 13 from Ref. [18] shows both their calculation (denoted "This work") and other results. Their result was in reasonable agreement with other exclusive decay calculations, but with smaller error. However, the black diamond labeled BLNP, which comes from inclusive decays, is larger. There have been no more recent published results for the $B \rightarrow \pi \ell v$ form factors. The FLAG report summarizes form factor results and their comparison with experiment (which determines $\left|V_{u b}\right|$ ). We will not reproduce those graphs here, but Fig. 14 shows the FLAG summary plot for $\left|V_{u b}\right|$, which considers both leptonic and semileptonic decays, and we quote the average value $3.73(14) \times 10^{-3}$, which is a 3.8\% error. The BaBar and Belle leptonic decay results do not agree very well, so FLAG reports results based on each experiment and their average. The experimental error is dominant for all values of $N_{f}$. Only for $N_{f}=2+1$ are there semileptonic form factors from lattice QCD. The lattice QCD form factor and experimental measurements from BaBar and Belle are jointly fit to a $z$-expansion with $\left|V_{u b}\right|$ as a free parameter. One sees that currently the error from semileptonic determination of $\left|V_{u b}\right|$ is smaller than the determination from leptonic decays.

An interesting story has emerged over the last several years regarding $V_{c b}$. In this case, both semileptonic decays $B \rightarrow D \ell v$ and $B \rightarrow D^{*} \ell v$ have been measured. The inclusive measurements also have been used to determine $\left|V_{c b}\right|$. In 2014, Ref. [19] updated the form factor for $B \rightarrow D^{*}$ at zero-recoil, and used the experimental average from HFLAV [20] (then using a different acronym) to obtain $\left|V_{c b}\right|=\left(39.04 \pm 0.49_{\text {expt }} \pm 0.49_{Q C D} \pm 0.19_{Q E D}\right) \times 10^{-3}$. The inclusive value from Gambino and Schwanda [21], was $(42.42 \pm 0.86) \times 10^{-3}$, which differs by $3.0 \sigma$ from the exclusive value. The next year, results for the $B \rightarrow D$ semileptonic decay became available [22], which gave 


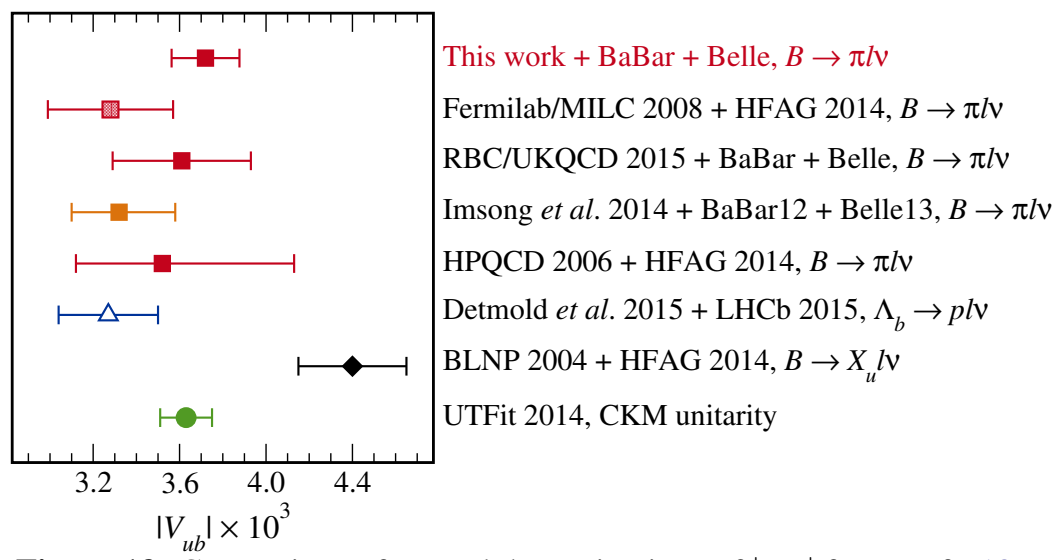

Figure 13: Comparison of several determinations of $\left|V_{u b}\right|$ from Ref. [18].

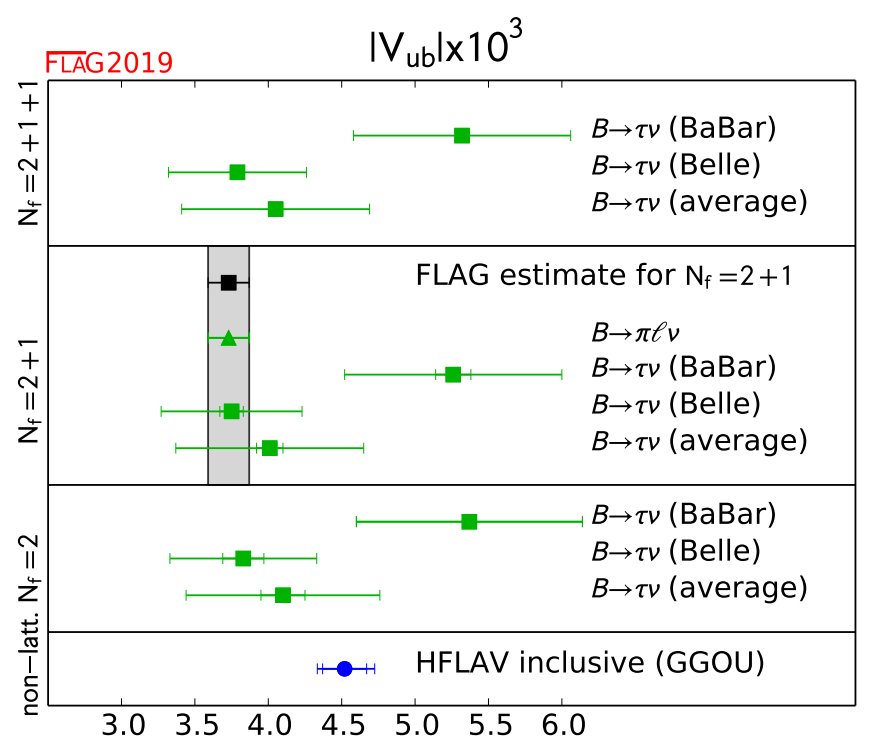

Figure 14: FLAG summary plot for $\left|V_{u b}\right|$ from Ref. [2].

a slightly larger value of $\left|V_{c b}\right|$, but with larger errors. Updated results from Belle became available for $B \rightarrow D$ which Bigi and Gambino [23] analyzed using the BGL form factor parametrization [24]. They found $\left|V_{c b}\right|=(40.49 \pm 0.97) \times 10^{-3}$. The next year, two groups looked at new Belle data [25] for $B \rightarrow D^{*}$. Both Bigi, Gambino, Schacht [26]; and Grinstein and Kobach [27] found about a $10 \%$ difference when switching between CLN [28] and BGL parametrizations of the form factors. At the time of FPCP 2018, I thought the so-called $V_{c b}$ puzzle was largely resolved [29] as the exclusive value of $\left|V_{c b}\right|$ using the BGL parametrization was quite compatible with the inclusive value, and the difference between CLN parametrized value and the inclusive value was only $1.36 \sigma$. However, the situation subsequently became more murky. At first, Belle [30] put out a preprint in September, 2018, that supported the notion that there is a 10\% difference when switching between CLN and BGL. Then, in April, 2019, version 3 of that preprint found that CLN and BGL parametrizations are quite compatible. A month earlier, an analysis from BaBar [31], using an unbinned fit, an angu- 
lar analysis, the BGL parametrization found $\left|V_{c b}\right|=(38.36 \pm 0.90) \times 10^{-3}$. This result is in good agreement with previous values of $\left|V_{c b}\right|$ based on exclusive decays.

More details can be found in Andrew Lytle's review in these proceedings, and in a recent paper by Gambino, Jung, and Schacht [33]. After performing a number of fits to Belle data, the latter find about a $2 \sigma$ difference between exclusive and inclusive values for $\left|V_{c b}\right|$. Thus, the puzzle remains until there are improvements in the lattice QCD calculations and the experiments. The form factor calculations will likely be improved by four competing groups: FNAL/MILC, HPQCD, JLQCD, and LANL/SWME. The interested reader should check the proceedings for their contributions. Figure 15 displays current results using either BGL or CLN parametrizations.
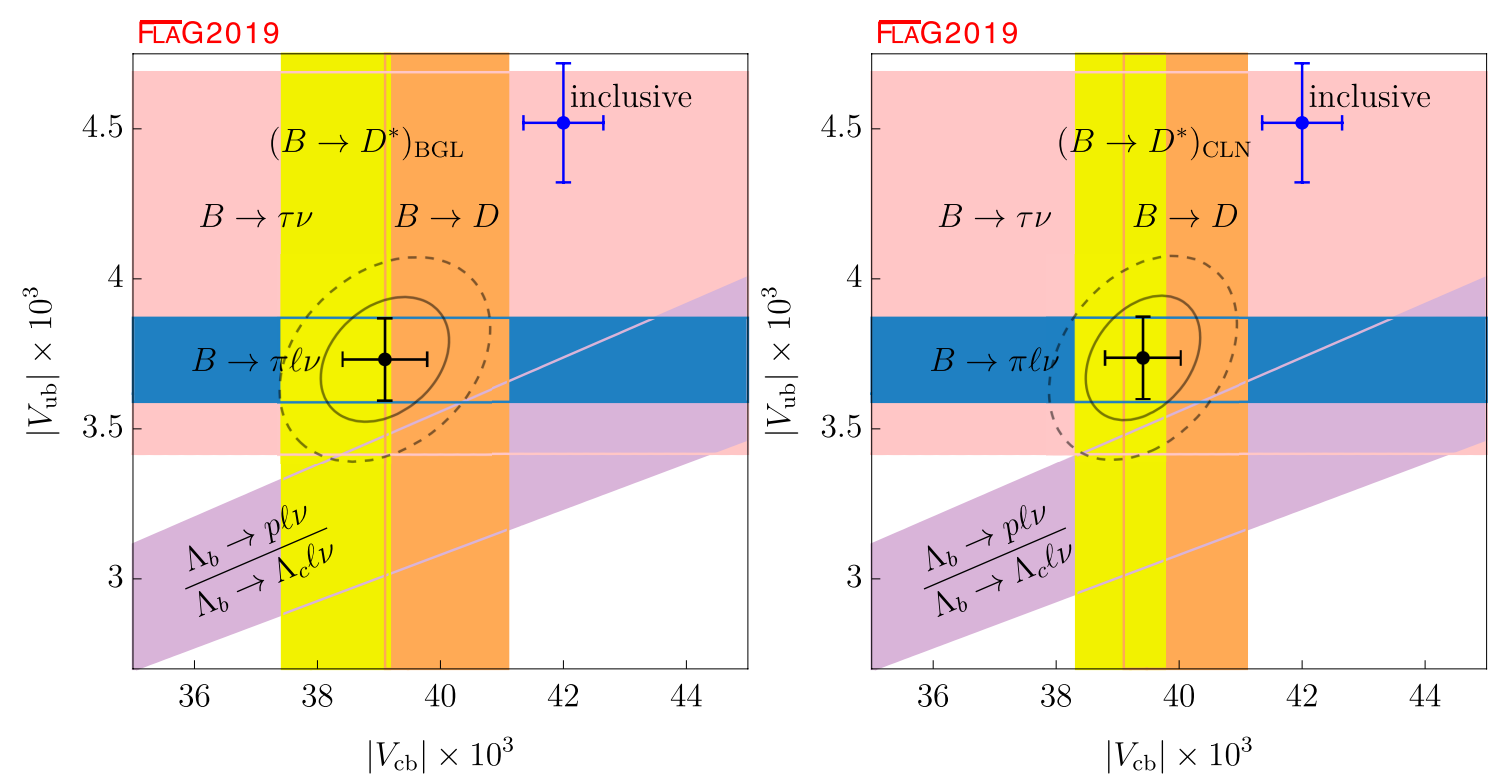

Figure 15: FLAG has summarized recent results for $\left|V_{u b}\right|$ and $\left|V_{c b}\right|$ [2]. On the left, the BGL parametrization for the $B \rightarrow D^{*}$ form factor is used. On the right, CLN is used. Further details may be found in Ref. [2].

\section{Third Row}

Neutral B meson mixing is a loop level process that depends upon $V_{t d}$ or $V_{t s}$ depending on whether we consider $B$ or $B_{s}$ meson mixing. Figure16 from a recent paper by the Fermilab Lattice and MILC Collaborations [34] shows the 1-loop diagrams responsible for neutral $B$ meson mixing in the standard model. The line labeled $q$ can either be a $d$ or $s$ quark. Both the mass difference and lifetime difference of the two resulting eigenstates are measured in experiments. A CP violating phase is also determined. A short distance expansion of the loops results in an effective weak Hamiltonian involving 4-quark operators. Because of the GIM and loop suppression of the mixing, this is a good place to look for BSM physics, which results in either operators that do not appear in 

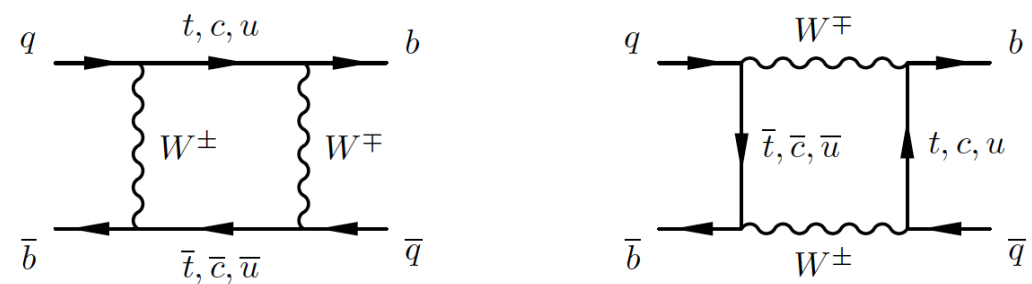

Figure 16: Box diagrams that contribute to neutral $B$ meson mixing from Ref. [34].

the standard model effective Hamiltonian, or modification of the coefficient of the standard model operator.

Briefly, it is conventional to define the effective Hamiltonian in terms of eight operators, where only the first one appears in the standard model.

$$
\mathscr{H}_{\mathrm{eff}}=\sum_{i=1}^{5} C_{i} \mathscr{O}_{i}^{q}+\sum_{i=1}^{3} \tilde{C}_{i} \tilde{\mathscr{O}}_{i}^{q}
$$

The first operator is given by

$$
\mathscr{O}_{1}^{q}=\bar{b}^{\alpha} \gamma_{\mu} L q^{\alpha} \bar{b}^{\beta} \gamma_{\mu} L q^{\beta}
$$

and its matrix element is conventionally defined as [35]

$$
\left\langle\mathscr{O}_{1}^{q}\right\rangle(\mu)=c_{1} f_{B_{q}}^{2} M_{B_{q}}^{2} B_{B_{q}}^{(1)}(\mu),
$$

where the last factor is called the bag parameter and would be 1 in the vacuum saturation approximation. It is also convenient to define a renormalization-group-invariant bag parameter $\hat{B}_{B_{q}}^{(1)}$ :

$$
\hat{B}_{B_{q}}^{(1)}=\alpha_{s}(\mu)^{-\gamma_{0} /\left(2 \beta_{0}\right)}\left[1+\frac{\alpha_{s}(\mu)}{4 \pi}\left(\frac{\beta_{1} \gamma_{0}-\beta_{0} \gamma_{1}}{2 \beta_{0}^{2}}\right)\right] B_{B_{q}}^{(1)}(\mu)
$$

In the standard model, we can express the difference in the masses of the mixing eigenstates as

$$
\Delta M_{q}=\frac{G_{F}^{2} m_{W}^{2} M_{B_{q}}}{6 \pi^{2}} S_{0}\left(x_{t}\right) \eta_{2 B}\left|V_{t q}^{*} V_{t b}\right|^{2} f_{B_{q}}^{2} \hat{B}_{B_{q}}^{(1)}
$$

Details can be found in Ref. [34]. The key point is that the measurable mass difference is proportional to $\left|V_{t q}\right|^{2}$ and the bag parameter (or that bag parameter multiplied by the square of the decay constant). Figure 17 contains the FLAG [2] summary of $f_{B_{d}} \sqrt{\hat{B}_{B_{d}}}$ and $f_{B_{s}} \sqrt{\hat{B}_{B_{s}}}$. There are three results that are averaged for $N_{f}=2+1$ and one result for $N_{f}=2$. FLAG does not calculate the impact of these results on the determination of the CKM matrix elements $V_{t d}$ and $V_{t s}$; however, Ref. [34] does. That paper also contains results for BSM operators.

Using experimental results for $B_{(s)}$, mixing, it is found in Ref. [34] that

$$
\begin{aligned}
\left|V_{t d}\right| & =8.00(33)(2)(3)(8) \times 10^{-3}, \\
\left|V_{t s}\right| & =39.0(1.2)(0.0)(0.2)(0.4) \times 10^{-3}, \\
\left|V_{t d} / V_{t s}\right| & =0.2052(31)(4)(0)(10),
\end{aligned}
$$




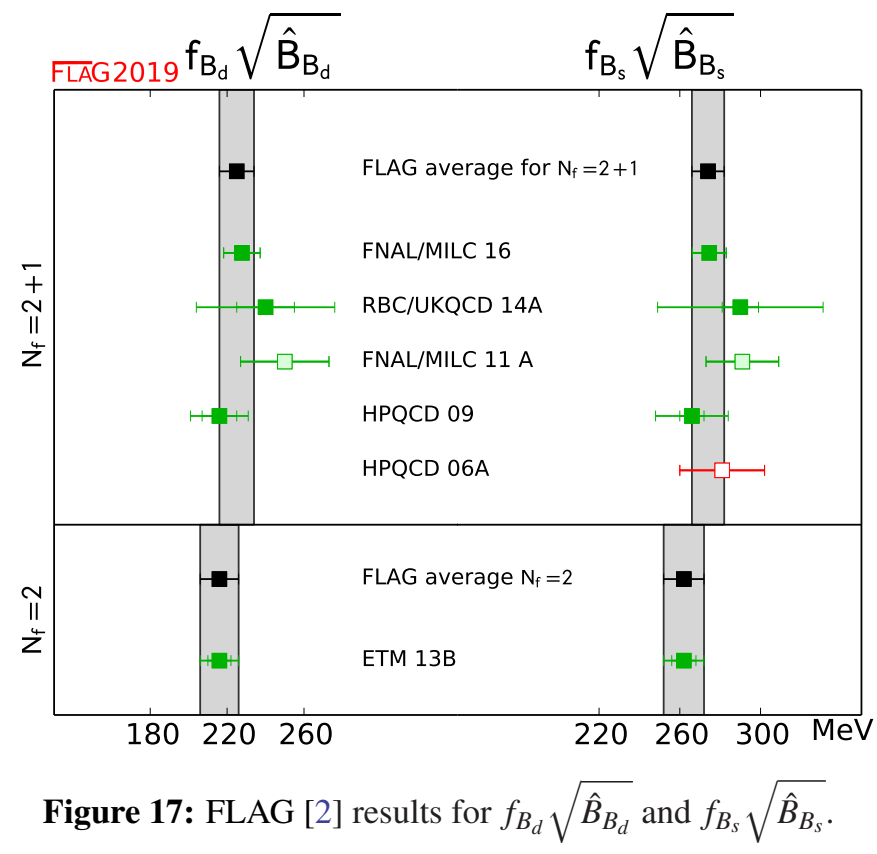

where in each case the first error is from the error in the lattice mixing matrix element, the second error comes from the error in the experimental mass difference, the third error comes from errors in parameters used in Eq. (6.5), and the final error is from the lack of charm quarks in the sea. One can see that the lattice errors are dominant, even in the ratio of CKM matrix elements in which the hadronic uncertainties are suppressed. The errors on each quantity are $4.3 \%, 3.2 \%$, and $1.6 \%$, from Eq. 6.6 to Eq. 6.8, respectively.

\section{Summary}

Table 1 shows the value of the magnitude of each CKM matrix element except $V_{t b}$. The second column has the value, and the third, the percentage error. We see that we have sub percent accuracy in the first row, but the other elements have errors ranging from $1.4 \%$ to $4.3 \%$. The final column indicates the source of each result. This is particularly pertinent for $\left|V_{u d}\right|$ and $\left|V_{u s}\right|$ as we have already remarked on the tensions with unitarity for the first row, and what happens if one considers one leptonic and semileptonic decays without input from nuclear $\beta$-decay on $\left|V_{u d}\right|$.

\section{Prospects}

Yogi Berra is purported to have said, "It is tough to make predictions, especially about the future." I trust you will keep that in mind as you read this section.

We have seen that in a number of quantities the theory error is the limiting factor in determining a CKM matrix element. Even in cases for which the experimental and theoretical errors are currently comparable, we can expect that BESIII, Belle II, and LHCb will reduce the experimental errors. The Belle II Physics Book [36] details what is expected to be accomplished at Belle II in many topics. In order to improve the future precision of CKM matrix elements, it is essential for 


\begin{tabular}{lccc}
\hline \hline Quantity & value & percentage error & Comment \\
\hline$\left|V_{u d}\right|$ & $0.9737(16)$ & 0.16 & FLAG (with unitarity) \\
$\left|V_{u d}\right|$ & $0.9669(34)$ & 0.35 & FNAL/MILC (K $\mathrm{K}_{12} \& \mathrm{~K}_{13}$ ) \\
$\left|V_{u s}\right|$ & $0.2249(7)$ & 0.31 & FLAG (with unitarity) \\
$\left|V_{u s}\right|$ & $0.22333(61)$ & 0.27 & FNAL/MILC (K 12 \& K K $\left._{13}\right)$ \\
$\left|V_{c d}\right|$ & $0.2219(43)$ & 1.9 & FLAG (2+1+1) \\
$\left|V_{c s}\right|$ & $1.002(14)$ & 1.4 & FLAG (2+1+1) \\
$\left|V_{u b}\right| \times 10^{3}$ & $3.76(14)$ & 3.7 & FLAG (BGL, combined) \\
$\left|V_{c b}\right| \times 10^{3}$ & $41.47(70)$ & 1.7 & FLAG (BGL, combined) \\
$\left|V_{t d}\right| \times 10^{3}$ & $8.00(34)$ & 4.3 & FNAL/MILC \\
$\left|V_{t s}\right| \times 10^{3}$ & $39.0(1.3)$ & 3.2 & FNAL/MILC
\end{tabular}

Table 1: Summary of CKM matrix elements determined with input from lattice QCD.

increased theoretical precision. It is assumed in this work that there will be a factor of five improvement in errors from lattice QCD in ten years. Figure 85 of Ref. [36] predicts the error on $V_{u b}$ from the study $B \rightarrow \pi \ell v$, taking into account both increased integrated luminosity and presumed improvement in lattice QCD precision in five and ten years. The figure makes clear how important it is to improve our calculations to make the best use of data coming from Belle II. Failure to improve the theoretical input could increase the error on $V_{u b}$ by a factor of two or more.

There has also been a recent report on future prospects of the LHC [37] where we can look forward to both the high-luminosity and higher energy enhancements of the machine. This report assumes a factor of three improvement in theoretical precision. A white paper from USQCD on flavor physics [38] will also interest the reader. We see that there is both a need and an expectation that the lattice QCD community will continue to improve our calculations.

\section{Conclusions}

Over the past few years, there has been very significant progress in using lattice QCD to calculate standard model parameters such as quark masses, the strong coupling $\alpha_{s}$, and matrix elements need to determine the CKM matrix. A number of quantities are now available at the subpercent level, and we expect the precision to increase by a factor of 3-5 over the next 5-10 years for form factors. Thus, the interplay between theory and experiment will provide more and more stringent tests of the Standard Model (and, perhaps, evidence of new physics). We are getting to the level of precision at which electromagnetic corrections are important. The Rome-Southampton group has shown leadership in the area and their work was presented in the parallel sessions.

Finally, BESIII, Belle II, and LHCb all have a large role to play in the future of flavor physics. I, for one, can hardly wait for their new results!

\section{Acknowledgments}

I am grateful to the local organizers and the International Advisory Committee for offering me the opportunity to give this talk. I owe a great debt to my friends and collaborators in the 
Fermilab Lattice and MILC Collaborations. I am thankful to all the members of the Flavour Lattice Averaging Group, whose work I have relied upon. In particular, working with the other members of the two working groups that deal with decay of hadrons containing charm or bottom quarks, and $B$ meson mixing has been a pleasure. Thanks to Zech Gelzer for preparing Fig. 12. Finally, this work was supported by the U.S. Department of Energy through grant DE-SC0010120.

\section{References}

[1] S. Aoki et al., Eur. Phys. J. C 77, no. 2, 112 (2017) doi:10.1140/epjc/s10052-016-4509-7 [arXiv:1607.00299 [hep-lat]].

[2] S. Aoki et al. [Flavour Lattice Averaging Group], arXiv:1902.08191 [hep-lat].

[3] W. J. Marciano, Phys. Rev. Lett. 93, 231803 (2004) doi:10.1103/PhysRevLett.93.231803 [hep-ph/0402299].

[4] M. Tanabashi et al. [Particle Data Group], Phys. Rev. D 98, no. 3, 030001 (2018). doi:10.1103/PhysRevD.98.030001

[5] M. Moulson, POS CKM 2016, 033 (2017) doi:10.22323/1.291.0033 [arXiv:1704.04104 [hep-ex]].

[6] A. Bazavov et al., Phys. Rev. D 98, no. 7, 074512 (2018) doi:10.1103/PhysRevD.98.074512 [arXiv:1712.09262 [hep-lat]].

[7] A. Bazavov et al. [Fermilab Lattice and MILC Collaborations], Phys. Rev. D 99, no. 11, 114509 (2019) doi:10.1103/PhysRevD.99.114509 [arXiv:1809.02827 [hep-lat]].

[8] M. Artuso et al. [CLEO Collaboration], Phys. Rev. Lett. 95, 251801 (2005) doi:10.1103/PhysRevLett.95.251801 [hep-ex/0508057].

[9] C. Aubin et al., Phys. Rev. Lett. 95, 122002 (2005) doi:10.1103/PhysRevLett.95.122002 [hep-lat/0506030].

[10] M. Ablikim et al. [BESIII Collaboration], Phys. Rev. Lett. 122, no. 7, 071802 (2019) doi:10.1103/PhysRevLett.122.071802 [arXiv:1811.10890 [hep-ex]].

[11] Y. S. Amhis et al. [HFLAV Collaboration], arXiv:1909.12524 [hep-ex].

[12] V. Lubicz et al. [ETM Collaboration], Phys. Rev. D 96, no. 5, 054514 (2017) Erratum: [Phys. Rev. D 99, no. 9, 099902 (2019)] doi:10.1103/PhysRevD.96.054514, 10.1103/PhysRevD.99.099902 [arXiv:1706.03017 [hep-lat]].

[13] H. Na, C. T. H. Davies, E. Follana, G. P. Lepage and J. Shigemitsu, Phys. Rev. D 82, 114506 (2010) doi:10.1103/PhysRevD.82.114506 [arXiv:1008.4562 [hep-lat]].

[14] H. Na, C. T. H. Davies, E. Follana, J. Koponen, G. P. Lepage and J. Shigemitsu, Phys. Rev. D 84, 114505 (2011) doi:10.1103/PhysRevD.84.114505 [arXiv:1109.1501 [hep-lat]].

[15] L. Riggio, G. Salerno and S. Simula, Eur. Phys. J. C 78, no. 6, 501 (2018) doi:10.1140/epjc/s10052-018-5943-5 [arXiv:1706.03657 [hep-lat]].

[16] W. Detmold, C. Lehner and S. Meinel, Phys. Rev. D 92, no. 3, 034503 (2015) doi:10.1103/PhysRevD.92.034503 [arXiv:1503.01421 [hep-lat]]. 
[17] D. Du, A. X. El-Khadra, S. Gottlieb, A. S. Kronfeld, J. Laiho, E. Lunghi, R. S. Van de Water and R. Zhou, Phys. Rev. D 93, no. 3, 034005 (2016) doi:10.1103/PhysRevD.93.034005 [arXiv:1510.02349 [hep-ph]]. The introduction of this paper summarizes the issues and provides many references.

[18] J. A. Bailey et al. [Fermilab Lattice and MILC Collaborations], Phys. Rev. D 92, no. 1, 014024 (2015) doi:10.1103/PhysRevD.92.014024 [arXiv:1503.07839 [hep-lat]].

[19] J. A. Bailey et al. [Fermilab Lattice and MILC Collaborations], Phys. Rev. D 89, no. 11, 114504 (2014) doi:10.1103/PhysRevD.89.114504 [arXiv:1403.0635 [hep-lat]].

[20] Y. Amhis et al. [Heavy Flavor Averaging Group], arXiv:1207.1158 [hep-ex].

[21] P. Gambino and C. Schwanda, Phys. Rev. D 89, no. 1, 014022 (2014) doi:10.1103/PhysRevD.89.014022 [arXiv:1307.4551 [hep-ph]].

[22] J. A. Bailey et al. [MILC Collaboration], Phys. Rev. D 92, no. 3, 034506 (2015) doi:10.1103/PhysRevD.92.034506 [arXiv:1503.07237 [hep-lat]].

[23] D. Bigi and P. Gambino, Phys. Rev. D 94, no. 9, 094008 (2016) doi:10.1103/PhysRevD.94.094008 [arXiv:1606.08030 [hep-ph]].

[24] C. G. Boyd, B. Grinstein and R. F. Lebed, Phys. Rev. D 56, 6895 (1997) doi:10.1103/PhysRevD.56.6895 [hep-ph/9705252].

[25] A. Abdesselam et al. [Belle Collaboration], arXiv:1702.01521 [hep-ex].

[26] D. Bigi, P. Gambino and S. Schacht, Phys. Lett. B 769, 441 (2017) doi:10.1016/j.physletb.2017.04.022 [arXiv:1703.06124 [hep-ph]].

[27] B. Grinstein and A. Kobach, Phys. Lett. B 771, 359 (2017) doi:10.1016/j.physletb.2017.05.078 [arXiv:1703.08170 [hep-ph]].

[28] I. Caprini, L. Lellouch and M. Neubert, Nucl. Phys. B 530, 153 (1998) doi:10.1016/S0550-3213(98)00350-2 [hep-ph/9712417].

[29] S. Gottlieb, Springer Proc. Phys. 234, 235 (2019) doi:10.1007/978-3-030-29622-3_32 [arXiv:1812.11211 [hep-lat]].

[30] E. Waheed et al. [Belle Collaboration], Phys. Rev. D 100, no. 5, 052007 (2019) doi:10.1103/PhysRevD.100.052007 [arXiv:1809.03290 [hep-ex]].

[31] J. P. Lees et al. [BaBar Collaboration], Phys. Rev. Lett. 123, no. 9, 091801 (2019) doi:10.1103/PhysRevLett.123.091801 [arXiv:1903.10002 [hep-ex]].

[32] A. Lytle, POS LATTICE 2019, 228 (2020).

[33] P. Gambino, M. Jung and S. Schacht, Phys. Lett. B 795, 386 (2019) doi:10.1016/j.physletb.2019.06.039 [arXiv:1905.08209 [hep-ph]].

[34] A. Bazavov et al. [Fermilab Lattice and MILC Collaborations], Phys. Rev. D 93, no. 11, 113016 (2016) doi:10.1103/PhysRevD.93.113016 [arXiv:1602.03560 [hep-lat]].

[35] F. Gabbiani, E. Gabrielli, A. Masiero and L. Silvestrini, Nucl. Phys. B 477, 321 (1996) doi:10.1016/0550-3213(96)00390-2 [hep-ph/9604387].

[36] E. Kou et al. [Belle-II Collaboration], arXiv:1808.10567 [hep-ex].

[37] A. Cerri et al., arXiv:1812.07638 [hep-ph].

[38] C. Lehner et al. [USQCD Collaboration], Eur. Phys. J. A 55, no. 11, 195 (2019) doi:10.1140/epja/i2019-12891-2 [arXiv:1904.09479 [hep-lat]]. 